\title{
3D Confocal Imaging of Pollen
}

\author{
Stanislav Vitha*, Vaughn M. Bryant**, Amen Zwa***, Andreas Holzenburg****** \\ * Microscopy and Imaging Center, Texas A\&M University, College Station, TX 77843 \\ ** Department of Anthropology, Texas A\&M University, College Station, TX 77843 \\ *** Gannon Technologies Group, 1000 North Payne Street, Alexandria, VA 22314 \\ **** Department of Biology \& Department of Biochemistry and Biophysics, Texas A\&M \\ University, College Station, TX 77843 USA
}

The study of pollen is highly useful in paleoecology, paleontology, archeology, and forensics. The outer shell of a pollen grain bears characteristic features that help in determining the species or genus of origin. To view the pollen grain from several angles, the sample is mounted in glycerol and rolled on the microscopic slide by pushing the coverslip. For teaching, training and also for establishing databases of pollen images, it is desirable to obtain three-dimensional datasets that then allow rotation, viewing and reslicing in the computer. We have employed sample preparation and mounting techniques that allow single-photon $3 \mathrm{D}$ confocal imaging of even relative large grains (more than $50 \mu \mathrm{m}$ in diameter) without excessive loss of resolution towards the bottom of the 3D datasets. Two key elements of our technique are pollen extraction and refractive index matching.

Sample preparation: Fresh or dried pollen was processed using a modified acetolysis method [1] in order to remove the cellular contents and debris, and to isolate the pollen outer shell. This eliminated a substantial amount of light-scattering and light-absorbing material from the lumen of pollen grains, which under normal circumstances causes serious difficulties in single-photon confocal microscopy. As a result of the acetolysis method, the pollen grain has been reduced to the resistant outer shell that carries the unique structural features used in identification. The pollen suspended in glacial acetic acid was then gradually re-hydrated and infiltrated with 2,2'-thiodiethanol (TDE), a mounting medium for high-resolution microscopy [2]. The final concentration was $97 \% \mathrm{v} / \mathrm{v}$ TDE in phosphate buffered saline. The rehydration and infiltration was accelerated by microwave irradiation for $1 \mathrm{~min}$ at $230 \mathrm{~W}$ in each step, using the Pelco Biowave (Ted Pella Inc., Redding, CA) laboratory microwave.

Confocal Microscopy: The Olympus FV1000 laser scanning confocal microscope with a 100x/1.4 oil immersion objective was used in photon counting mode with the confocal aperture set to 0.75 Airy units. Excitation wavelength was $488 \mathrm{~nm}$, emission bandpass was 500-600nm. The laser output was programmed to increase with imaging depth to compensate for the loss of signal. The voxel size was $65 \times 65 \times 130 \mathrm{~nm}$. The axial resolution of the objective under these conditions was previously measured as $418 \pm 43 \mathrm{~nm}$ (FWHM, using $100 \mathrm{~nm}$ fluorescent beads). Confocal datasets were further processed in MATLAB (The MathWorks Inc., Natick, MA) to correct for residual attenuation of the signal in the bottom of the stacks, and 3D surface rendering was performed using Osirix freeware (http://www.osirix-viewer.com).

Results: Acquisition times were between 20 and 50 min per stack, depending on the size of the pollen grain. Visual inspection of the stacks indicated little obvious deterioration of resolution with depth (Fig. 1). Surface rendering enabled realistic visualization of the pollen grain (Fig. 2). Further possible improvements in imaging conditions and post-processing are discussed. 
References

1. $\quad$ Erdtman, G., The acetolysis method. Svensk Botanisk Tidskrift, 1960 54: p. 561-564.

2. Staudt, T., M.C. Lang, R. Medda, J. Engelhardt, and S.W. Hell, 2,2'-thiodiethanol: a new water soluble mounting medium for high resolution optical microscopy. Microsc Res Tech, 2007. 70(1): p. 1-9.
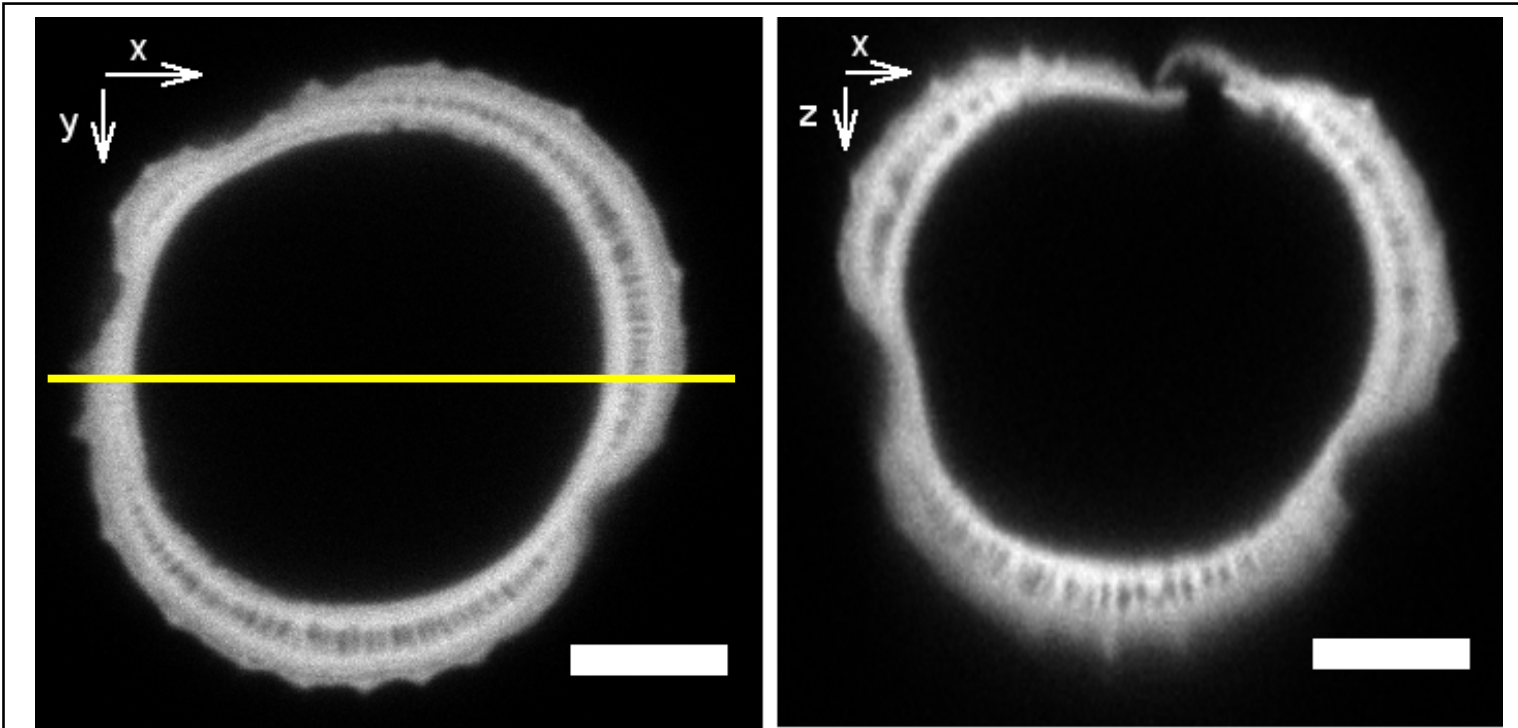

Figure 1. Confocal optical sections of Artemisia pollen grain. Left: XY view, a single optical section from the Z-stack. The yellow line indicates the position of an XZ section through the z-stack, shown in the right panel. Scale bar $=5 \mu \mathrm{m}$.
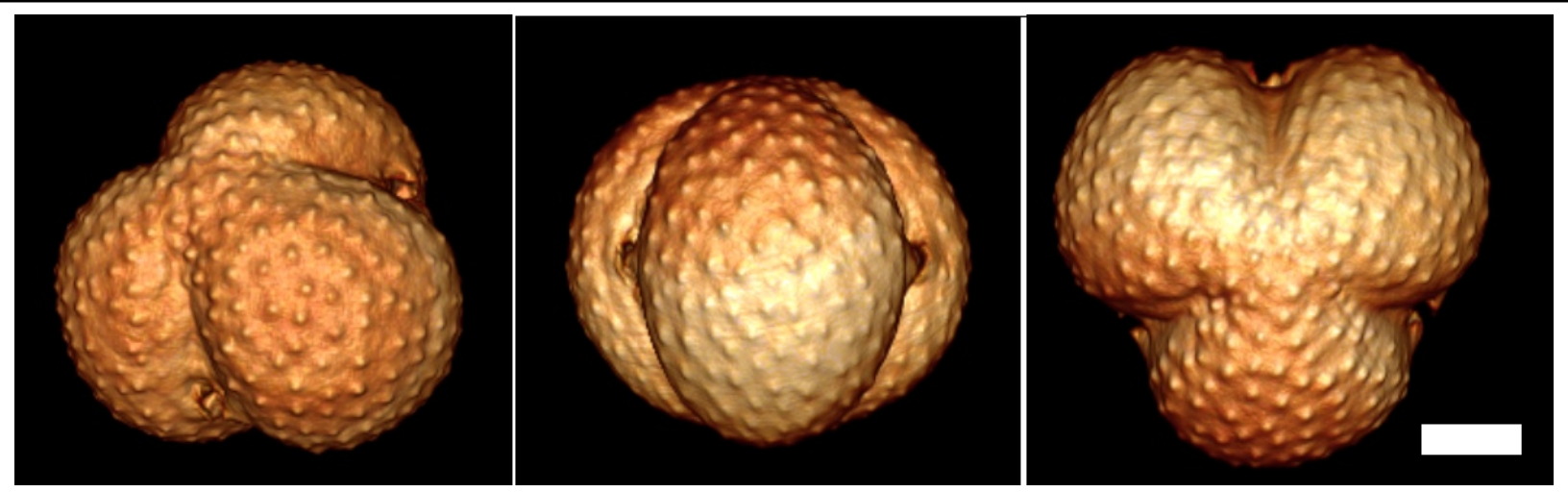

Figure 2. Surface rendering of Artemisia pollen grain, showing three different views generated from the same dataset. Scale bar $=5 \mu \mathrm{m}$. 